\title{
Actitudes y emociones: Pautas para el aprendizaje del idioma inglés en la educación superior intercultural
}

\author{
Attitudes and emotions: guidelines for the learning of English \\ language in higher intercultural education
}

Freddy Javier Chamorro Sady
Cesar Antonio Pérez Henríquez
${ }^{2}$
William Oswaldo Flores López ${ }^{3}$

\section{Resumen}

Este escrito proporciona pautas para el aprendizaje del idioma inglés en la educación superior intercultural, desde una perspectiva conceptual sobre las actitudes y emociones en el ámbito de educativo y su relación con aspectos cognitivos, afectivos, conativos y comportamental. El análisis se enmarca en estudio bibliográfico sobre el componente actitudinal y emocional enfocado en el aprendizaje del idioma inglés. Existen una gran responsabilidad por parte del estudiantado y más aún por el profesorado, en términos de cultivar actitudes y emociones en el proceso de aprendizaje de la competencia comunicativa del idioma inglés, además de incorporar tecnologías de la información y comunicación para incrementar el agrado, la motivación y la confianza hacia el aprender y comprender inglés.

Palabras clave: Actitudes, emociones, idioma inglés; aprendizaje, tecnología.

\section{Abstract}

This paper provides guidelines for learning the English language in intercultural higher education, from a conceptual perspective on attitudes and emotions in the field of education and its relationship with cognitive, affective, conative and behavioral aspects. The analysis is framed in a bibliographic study on the attitudinal and emotional component focused on the learning of the English language. There is a great responsibility on the part of the students and even more so by the teachers, in terms of cultivating attitudes and emotions in the learning process of the communicative competence of the English language, besides incorporating information and communication technologies to increase the liking, the motivation and confidence towards learning and understanding English.

\footnotetext{
1 Bachelor in Science of Education with majoring in English. Teacher at The University of the Autonomous Regions of the Nicaraguan Caribbean Coast-Nueva Guinea Campus. E-mail:: freddy.chamorro@uraccan.edu.ni ORCID: https://orcid.org/oooo-ooo1-9889-6500

2 Bachelor in Pedagogy. Consultant and Researcher. E-mail:: cp_henriquez69@yahoo.es

3 Doctor en Educación. PhD in Education. Research Professor at The University of the Autonomous Regions of the Nicaraguan Caribbean Coast. E-mail:: william.flores@uraccan.edu.ni ORCID: https://orcid.org/oooo-ooo2-1016-1620
} 
Key words: attitudes, emotions, English language, learning, technology.

\section{Introducción}

Las actitudes y emociones juegan un papel importante en el ámbito profesional, además muchos investigadores argumentan que la educación esta experimentando un giro hacia lo afectivo (Pekrun \& Linnenbrink-García, 2014; Uitto, Jokikokko \& Estola, 2015). En la educación superior de Nicaragua, existe una deserción del estudiantado universitario por factores como: emotivos, académicos, económicos, motivacionales, normativos, ambientes familiares, orientación profesional, absentismos por género (femenino), marginación cultural, étnico, lingüísticos, discapacidad y procesos de acompañamiento al estudiantado en su formación (Flores et al., 2016).

Nias (1996) señala que las emociones son fundamentales en el proceso de aprendizaje por dos razones: en primer lugar, el proceso educacional implica la interacción entre personas; en segundo lugar; porque la identidad personal y profesional de los profesores, en muchas ocasiones son inseparables y en el aula se convierten en factores de influencia en la autoestima y en el bienestar personal y social. El rol del docente implica una importante carga de trabajo emocional, tanto por lo que exige sensibilidad a las emociones ajenas como por lo que exige de manejar apropiadamente las emociones propias e impropias para facilitar y optimizar la calidad de relaciones interpersonales que caracterizan a las instituciones educativas (Casassús, 2007; Fernández-Berrocal \& Ruíz, 2008; y Hargreaves, 2000).

Teniendo de referencia lo anterior, el presente texto argumentativo brindará pautas sobre las actitudes y emociones hacia el aprendizaje del idioma inglés en la educación superior intercultural. Se trata de describir los elementos importantes sobre las actitudes y emociones en el aprendizaje del idioma inglés, sabiendo que, en la educación nicaragüense, la competencia del idioma inglés es asumida desde el aprendizaje de las cuatro habilidades básicas, es decir, leer, escribir, escuchar y hablar, así como el desarrollo de actitudes positivas hacia la diversidad lingüística propiciando sus ideas, emociones y sentimientos mediante la comprensión de un nuevo idioma (Canelo \& Acevedo, 2009).

\section{Reflexión educativa}

\section{Actitudes}

En la educación en general, las actitudes pueden considerarse uno de los aspectos psicológicos que han alcanzado más difusión. Este hecho puede asociarse a la calidad de desarrollo del aprendizaje del idioma inglés en particular. Por ello es importante, en primera instancia, caracterizar el término de actitudes. Por tanto, iniciamos con la definición de Gil, Blanco y Guerrero (2005) de actitudes se conciben como una 


\section{REFLEXIONES EDUCATIVAS}

predisposición, con cierta carga emocional, que influye en la conducta; esta definición remarca tres componentes básicos de la actitud: la cognición o creencia sobre objeto de la misma, el afecto o carga evaluativa de dichas creencias y una intención de conducta en relación a dicho objeto. Por su parte Rokeach (1968) citado por Cardoso y Vanegas (2012), opinó que las actitudes son organizaciones de creencias relativamente permanentes que predisponen a responder de un modo preferencial ante un objeto o situación. En cambio, Likert (1976), afirmó que las actitudes son disposiciones hacia una acción manifiesta. Sobre la base de estas afirmaciones se puede derivar las siguientes definiciones de actitud:

- Gairín (1990) indica que las actitudes son instancias que predisponen y dirigen al sujeto sobre la realidad, filtran las percepciones y orientan el pensamiento para adaptarlo al contexto.

- En Clemente (1995) se definen las actitudes como predisposiciones de valoración que son emitidas por los sujetos, así también como sentimientos positivos o negativos que están asociados con algún objeto psicológico que conduce al sujeto a actuar y expresarse según ellos, es decir en cada uno de sus actos y opiniones.

- En Anastasi y Urbina (1998) las definen como una predisposición evaluativa (positiva o negativa) que determina las intenciones personales e influye en el comportamiento.

- En Gómez-Chacón (2000) se dice que las actitudes son un campo de creencias, sentimientos y estado de ánimo que transciende el dominio de la cognición.

- En Guerrero, Blanco y Vicente (2002) las conceptualizan como una predisposición conformada de acuerdo una serie de convicciones y sentimientos que hacen que el sujeto reacción con sus creencias y sentimientos.

De acuerdo con estos conceptos, se pueden sintetizar que las actitudes vienen a ser predisposiciones comportamentales u orientaciones afectivas que un sujeto adquiere y que acompaña con una reacción valorativa y evaluativa, manifestada a través del agrado o desagrado, hacia algún objeto, sujeto o situación. Es decir, son predisposiciones o juicios valorativos o evaluativos, favorables o desfavorables, que determinan las intenciones personales de los sujetos y son capaces de influir sus comportamientos o acciones frente al objeto, sujeto o situación (Martínez, 2008).

Las actitudes pueden expresarse mediante factores como: ideas, percepciones, gustos, preferencias, opiniones, creencias, emociones, sentimientos, comportamientos y tendencias a actuar. Tales factores han sido estudiados por autores como Auzmendi (1992) y Caballero, Guerrero y Blanco (2014) y fueron organizado en funcion de componentes o dimensiones actitudinales, que a continuación se detallan: 
1. Cognitivo (el conocer/ el saber). Se corresponde con la carga de información y la experiencia adquirida por el sujeto, en correspondencia al objeto de su actitud. Se manifiesta o expresa mediante percepciones, ideas, opiniones, concepciones y creencias a partir de las cuales el sujeto se coloca a favor o en contra de la conducta esperada. La predisposición a actuar de manera preferencial hacia el objeto, persona o situación sujeta a este componente. En Auzmendi (1992) se dice que estos aspectos poseen algunas características que los diferencian de los elementos psíquicos. Estos son:

- Fijación. El componente cognitivo de las actitudes está arraigado en el psiquismo humano. Se caracteriza por su carácter fijo y estable, hecho que lo diferencia de la mera opinión.

- Singularidad. Se trata de un elemento simple puesto que se re ere a un único objeto, persona o situación.

- No son valores. Los valores se caracterizan por su alta abstracción y amplia predicabilidad.

- Toma de consciencia. Estos componentes no siempre se expresan en forma consciente.

2. Afectivo (la emoción/ el sentir). Este componente se pone de manifiesto por medio de las emociones y los sentimientos de aceptación o de rechazo que el sujeto activa motivacionalmente ante la presencia del objeto, persona o situación que genera dicha actitud. También se remite al valor que el sujeto le atribuye.

3. Conativo o Intencional (la intención). Es expresada por los sujetos mediante su inclinación voluntaria de hacer una acción. Está constituido por predisposiciones, predilecciones, preferencias, tendencias o intenciones de actuar de una forma específica ante el objeto, según las orientaciones de las que existen. La tendencia de actuar, favorable o desfavorable, se pone de manifiesto a través de las acciones del sujeto ante el objeto de su actitud.

4. Comportamental (comportamiento). Constituye la conducta observable, propiamente dicha, la cual, según Postic y De Ketele (1992), será concebida como un conjunto de comportamientos. Es decir, las actitudes no son únicamente creencias sobre un objeto determinado acompañado de un afecto respecto al mismo, sino disposiciones a reaccionar ante un estímulo (Auzmendi, 1992).

Con referencia a lo anterior, en Martínez (2008) se exponen una serie de singularidades sobre actitudes:

- Implican una evaluación hacia algo o alguien que se materializa mediante una emoción de juicios valorativos, pudiendo referirse a una o varias cosas, o a una o varias personas o situaciones.

- Suelen ser relativamente estables, determinan las intenciones personales e influyen en el comportamiento de los sujetos. 


\section{REFLEXIONES EDUCATIVAS}

- Actúan como motivadoras de la conducta y pueden constituirse en la única motivación para emprender el comportamiento y las acciones de los sujetos.

- Pueden expresarse mediante el lenguaje verbal y no verbal.

- No siempre tienen relación con la conducta trasmitida por el sujeto, pues, esto depende de otros factores intervinientes. Sin embargo, aunque no toda disposición dé a lugar a la acción correspondiente, suele, dentro de un umbral de la variable, presentar una cierta consistencia.

- No son observables en forma directa por lo que los que las investigan deben utilizar métodos alternativos para su determinación. Para lograr dicho fin, quienes observan las acciones y los comportamientos deben inferirlos. Esto es posible a través de la manifestación de las creencias, sentimientos, intenciones o conductas: verbalizaciones o expresiones de sentimiento acerca del objeto, por finalidad o evitación, tendencia o preferencia manifestada, etc.

- Conformar, junto con la formación teórica inicial de los profesores, sus experiencias y la reflexión sobre estas experiencias, lo que se denomina conocimiento profesional de los profesores.

En la línea de las ideas anteriores, se puede agregar que las actitudes son el resultado de un aprendizaje cultural y, en consecuencia, no son innatas y difieren en función del ambiente donde el sujeto las aprende (Martínez, 2008). Eso supone que, para analizarlas, suele considerarse el contexto donde se manifiestan y las interacciones que se producen entre los actores que protagonizan los comportamientos y las acciones debidas a ellas. Además, las actitudes juegan un papel que puede ser útil para la descripción, comprensión o explicación de una parte de la cultura de las aulas ya que, junto con las tradiciones, pueden ser perdurables y compartidas por grupos de personas y pueden ser trasmitidas de generación en generación (Myers, 1995).

\section{Emociones}

Salovey y Mayer (1990) definieron a la inteligencia emocional como una forma de inteligencia social que implica la capacidad de controlar nuestros propios sentimientos y los de otros, discriminar entre ellos y utilizar esa información para guiar nuestros pensamientos y acción. Desde esta perspectiva, las emociones son necesarias en el aprendizaje del idioma inglés, porque contribuyen al desarrollo de la competencia comunicativa, además que la inteligencia emocional es la capacidad para percibir, aplicar, comprender controlar o manejar sus propias emociones y sentimientos y entender el de los demás, para ser más efectivos en las relaciones interpersonales y que les permita vivir y convivir en un ambiente de paz. Así mismo, que la inteligencia emocional integra aptitudes personales (el autoconocimiento, la autorregulación y la motivación) y actitudes sociales (habilidades sociales). Todas ellas necesarias para conducirse y hacer frente a las situaciones adversas de la vida" (Canelo \& Acevedo, 2009, p. 71). 
Debido a esto, en los ambientes de aprendizajes abundan diversas emociones tales como el disfrute de los aprendizajes, la esperanza, el orgullo, la ansiedad, la vergüenza y el aburrimiento, además, que dichas emociones son sumamente importantes para la motivación de los aprendizajes, el desempeño y el desarrollo de la identidad (Pekrun, Frenzel, Goetz \& Perry, 2007). Las habilidades emocionales en el estudiantado tienen gran importancia para su desarrollo, no sólo académico, sino también personal. En el caso del profesorado tiene un doble significado, ya que deberán tener capacidad de identificar, comprender y regular, no sólo sus propias emociones, sino también las de sus discentes (Fernández-Berrocal y Extremera, 2002).

La educación emocional viene dada de forma implícita en numerosas ocasiones. Incluso las emociones han sido enseñadas antes de que el concepto de inteligencia emocional fuese desarrollado (Del Rosal, Moreno-Manso \& Bermejo, 2018). Investigaciones como la de Martínez y Villardón-Gallego (2015) muestran la necesidad de formar la competencias emocionales en la formación inicial de futuros maestros. Es por ello, que discentes que participan en programas de intervención emocional mostrarán mayor capacidad para identificar las emociones disponiendo de un lenguaje emocional más rico y diversos e incluso mayor satisfacción vital (Brígido et al., 2013). Además, llegarían a mejorar las estrategias de regulación emocional y autoconcepto desarrollando un mayor número de conductas asertivas con respecto a los individuos que no participan en dichos programas (Cazalla-Luna \& Molero, 2015).

Es por ello, que el componente actitudinal y emocional es organizado en el programa de idioma inglés como contenido a desarrollar valores y normas, con el propósito de fortalecer la función moral o ética de la educación. Pueden incluirse tres tipos de actitudes: actitudes hacia los contenidos conceptuales; actitudes y valores comunes a un conjunto de áreas o disciplinas, los que se ven como guías para el aprendizaje; y un conjunto de actitudes específicamente morales, ambientales que tiene carácter más transversal que específico de un área (Canelo \& Acevedo, 2009, p. 11).

\section{Conclusiones y perspectivas futuras}

En este ensayo se ha descrito una síntesis de los conceptos claves alrededor de las actitudes. Resulta relevante identificar las actitudes hacia el aprendizaje del idioma inglés en la educación superior intercultural y comunitaria, porque constituyen una información valiosa tanto para el diseño, planificación e implementación de los procesos de enseñanza por parte del profesorado, como para el desarrollo de los procesos formativos a generar en el estudiantado, quienes van a seguir interactuando con dicha disciplina no solo en el ámbito académico, sino también en el profesional.

Es claro, entonces, que hay una responsabilidad por parte del profesorado para desarrollar y afirmar las actitudes y emociones positivas en estudiantado, a través de estrategias que permitan beneficiar la predisposición favorable hacia el aprendizaje 


\section{REFLEXIONES EDUCATIVAS}

del idioma inglés con prácticas didácticas de la vida cotidiana que ayuden a mejorar la calidad de los aprendizajes, favoreciendo el desarrollo de las habilidades básicas: leer, escribir, hablar y escuchar. De tal manera que las actitudes ejercerán un efecto importante en el estudiantado.

Además, para incrementar la confianza, el agrado y la motivación en el aprendizaje del idioma inglés, el profesorado tiene que: integrar tecnologías que hagan mejorar las experiencias del aprendizaje del estudiantado; conocer y manejar bien las tecnologías disponibles para apoyar su función docente; utilizar las tecnologías para mejorar la gestión curricular en su ámbito de acción; usar las tecnologías como un medio de inclusión social, de atención a la diversidad, realizando una actuación ética y legal respeto de su uso y cuidando la salud y del medio ambiente; y finalmente, reconocer su responsabilidad para que el estudiantado tenga un aprendizaje cada vez más eficiente y actual, usando o incorporando las tecnologías y como docente asuma responsablemente su propio actualización y desarrollo profesional con las potencialidades que presentan las tecnologías para su quehacer profesional.

En definitiva, es necesario identificar las actitudes del estudiantado universitario y su relación con las variables género y etnia con la finalidad de diseñar, implementar y validar propuestas y metodologías que faciliten el aprendizaje de la competencia comunicativa del idioma inglés. Así como, construir perfiles motivacionales en la formación inicial del profesorado y así evitar la desmotivación del docente por factores actitudinales y emocionales. Todo lo anterior, con el propósito de ayudar a las instituciones educativas reducir la deserción del futuro estudiante de educación superior intercultural.

\section{Lista de referencia}

Anastasi, A., \& Urbina, S. (1998). Psicología. México: Prentice Hall.

Auzmendi, E. (1992). Las actitudes hacia la matemática-estadística en la enseñanza medias y universitaria. Bilbao: Mensajero.

Brígido, M., Borrachero, A. B., Bermejo, M. L., Mellado, V. (2013). Prospective primary teachers' self-efficacy and emotions in science teaching. European Journal of Teacher Education, 36(2), 200-217.

Caballero, A., Guerrero E., \& Blanco, L. (2014). Construcción y administración de un cuestionario para la evaluación de los afectos hacia las matemáticas. Campo Abierto, 33(1), 47-71.

Canelo, J., \& Acevedo, R. (2009). Programa de Estudio de Lengua Extranjera de Educación Secundaria (7mo, 8vo y gno grado). Managua: MINED. 
Cardoso E. O., \& Vanegas, E. A. (2012). Diagnóstico sobre la actitud hacia las matemáticas en tres posgrados en administración de empresa. Revista Electrónica Educared, 16(2), 237-253.

Casassús, J. (2007). La educación del ser emocional. Santiago de Chile: Editorial Cuarto.

Cazalla-Luna, N. y Molero, D. (2015). Inteligencia emocional percibida, disposición al optimismo-pesimismo, satisfacción vital y personalidad de docentes en su formación inicial. Revista de Investigación Educativa, 34(1), 241-258.

Clemente, M. (1995). Psicología Social: Métodos y Técnicas de Investigación. Madrid: Eduma.

Del Rosal, I., Moreno-Manso, J. M., \& Bermejo, M., L. (2018). Inteligencia emocional y rendimiento académico en futuros maestros de la Universidad de Extremadura. Revista de Curriculum y Formación del profesorado, 22(1), 257-275.

Fernández-Berrocal, P. y Extremera, N. (2002). La Inteligencia Emocional como una habilidad esencial en la escuela. Revista Iberoamericana de Educación, 29, 1- 6.

Fernández-Berrocal, P. y Ruiz, D. (2008). La inteligencia emocional en la educación. Revista Electrónica de Investigación Psicoeducativa, 6(2), 421-436.

Flores, W. O., \& Gutiérrez y Restrepo, E., León, O., Sarraipa, J., Pantoja, C., Merino, C., Calderón, D., Guinocchio, M., Rivera, M., Calderón, M., E., \& Boticario, J., G. (2016). Centros de Apoyo y Desarrollo Educativo Profesional para la observación y disminución de la deserción universitaria. Ciencia e Interculturalidad, 18(1), 48-62. DOI: http://dx.doi.org/10.5377/rci.v18i1.3049

Gairín, J. (1990). Las actitudes en educación. Un estudio sobre la educación matemática. Barcelona: Boixareau Universitaria

Gil, N., Blanco L., \& Guerrero, E. (2005). El dominio afectivo en el aprendizaje de las matemáticas. Una revisión de sus descriptores básicos. UNIÓN. Revista Iberoamericana de Educación Matemática, 2, 15-32.

Gómez-Chacón, I. (2000). Matemática Emocional: Los afectos en el aprendizaje Matemático. Madrid: Narcea.

Guerrero, E., Blanco, L., \& Vicente, F. (2002). Trastornos emocionales ante la educación matemática. España: Pirámide. 


\section{REFLEXIONES EDUCATIVAS}

Hargreaves, A. (2000). Mixed emotions: teachers' perceptions of their interactions with students. Teaching and Teacher Education, 16, 811-826. DOI: http://dx.doi. org/10.1016/So742-051X(oo)00028-7

Likert, R. (1976). Una técnica para la medición de actitudes. Buenos Aires: Nueva Visión.

Martínez, O. (2008). Actitud hacia la matemática. Sapiens: Revista Universitaria de Investigación, 9(1), 237-256.

Martínez, Z. y Villardón-Gallego, L. (2015). La imagen del profesor de educación secundaria en la formación inicial. Profesorado: Revista de Curriculum y Formación del profesorado, 19(1), 511-526.

Myers, D. (1995). Psicología Social. México: McGraw-Hill.

Nias, J. (1996). Thinking about feeling: the emotions in teaching. Cambridge Journal of Education, 26(3), 293-306. DOI: https://doi.org/10.1080/0305764960260301

Pekrun, R., \& Linnenbrink-García, L. (2014). International handbook of emotions in education. New York: Routledge.

Pekrun, R., Frenzel, A. C., Goetz, T. \& Perry, R. (2007). The control-value theory of achievement emotions. An integrative approach in education. In P. Schutz \& R. Pekrun. (Eds.), Emotion Educational Psychology (13-36). San Diego: Academic Press.

Postic, M., \& De Ketele, J., M. (1992). Observar situaciones educativas. Madrid: Narcea.

Salovey, P. Y Mayer, J. (1990). Emotional intelligence, imagination, cognition y personality, 9, 185-211.

Uitto, M., Jokikokko, K., \& Estola, E. (2015). Virtual special issue on teacher and emotions in teaching and teacher education (TATE) in 1985-2014. Teaching and Teacher Education, 1-12. 